\title{
Cross-cultural evidence of a space-ethnicity association in face categorisation
}

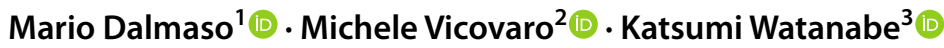

Accepted: 14 February 2022

(c) The Author(s) 2022

\begin{abstract}
According to a space-valence association, individuals tend to relate negatively- and positively-connoted stimuli with the left and right side of space, respectively. So far, only a few studies have explored whether this phenomenon can also emerge for social dimensions associated with facial stimuli. Here, we adopted a cross-cultural approach and conducted two experiments with the main aim to test whether a left-right space-valence association can also emerge for other- vs. own-race faces. Asian Japanese (Experiment 1) and White Italian (Experiment 2) participants engaged in a speeded binary classification task in which a central placed face had to be classified as either Asian or White. Manual responses were provided through a left- vs. right-side button. In both experiments, other-race faces elicited faster responses than own-race faces, in line with the well-documented other-race categorisation advantage. Moreover, evidence of an association between space and ethnic membership also arose and, interestingly, was similar in both groups. Indeed, Asian faces were responded to faster with the right-side key than with the left-side key, whereas response side had no effect for White faces. These results are discussed with regard to possible cross-cultural differences in group perception.
\end{abstract}

Keywords Other-race categorisation advantage $\cdot$ Space-valence association $\cdot$ Faces $\cdot$ Cross cultural $\cdot$ Social cognition

\section{Introduction}

As human beings, we are social in nature, and a substantial part of our daily life is typically spent establishing social relationships with others. When navigating social contexts, others' faces are likely the most frequently encountered and informative stimuli we can use to extract valuable information to regulate our own behaviour. For instance, others' eye-gaze direction can inform us about the allocation of their attentional resources over space, helping us detect meaningful objects within the surrounding environment (Dalmaso et al., 2020). Similarly, facial emotions can elicit either approach or avoidance behaviours in an observer (Adams et al., 2006). Invariant information can also be extracted

Mario Dalmaso

mario.dalmaso@unipd.it; mario.dalmaso@gmail.com

1 Department of Developmental and Social Psychology, University of Padova, Padova, Italy

2 Department of General Psychology, University of Padova, Padova, Italy

3 Faculty of Science and Engineering, Waseda University, Tokyo, Japan from others' faces, such as age and gender, which guide us in the construction of personal identities (Haxby et al., 2000).

During the last decades, the potential impact of ethnic membership on face processing mechanisms earned a central role in social cognition research. In this regard, several works have provided supporting evidence for the notion that own-race faces are processed differently from otherrace faces. This is well evidenced by two behavioural effects related to face recognition on the one hand and face categorisation on the other. As for face recognition, a well-known and robust phenomenon is the so-called other-race effect (ORE), consisting of higher accuracy in recognising ownrace face identities than identities associated with otherrace faces (e.g., Tanaka et al., 2004). Experimentally, the ORE has been widely explored and replicated, and it can be revealed through a variety of memory tasks consisting, for example, of exposing participants to a set of target faces that must then be recognised, among other previously unseen faces, in a subsequent testing phase (for a review see Hugenberg et al., 2010). As for face categorisation, literature shows an intriguing phenomenon known as the other-race categorisation advantage (ORCA), according to which other-race faces are categorised faster than own-race faces (e.g., Levin, 
1996; Valentine \& Endo, 1992). In this case, participants are typically asked to categorise own- vs. other-race faces by means of a speeded binary classification task (e.g., Ge et al., 2009; Woo et al., 2020). According to different theories and models, it seems plausible that both the ORE and ORCA would emerge as a consequence of common mechanisms underlying face processing, which would be mostly related to categorisation, motivational, and perceptual processes (Hugenberg et al., 2010). For instance, whereas own-race faces would be perceived and treated more holistically (i.e., as a whole), the processing of other-race faces would be mainly driven by analyses of single components (Tanaka et al., 2004), such as the eyes and the mouth, thus leading to less accurate recognition and faster categorisation of faces belonging to other ethnic groups (Zhao \& Bentin, 2011). Whereas the ORE has been the focus of numerous studies and debates, the ORCA has been much less investigated, thus leaving room for a number of open research questions.

From a methodological perspective, binary classification tasks - such as that employed to reveal the ORCA - are widely adopted and constitute a standard tool in experimental psychology to unveil a variety of mechanisms underlying cognition (Nickerson, 1972). An important aspect to consider when such tasks are used is the presence of potential overlapping between stimulus properties and response location. More specifically, literature clearly shows that if a perceptual or conceptual overlap exists between a given stimulus and space (e.g., left or right), then the presentation of that stimulus could influence the behavioural response according to the location of the response key. In this regard, a classic phenomenon due to a stimulus-response perceptual overlapping is the Simon effect (Simon \& Rudell, 1967) in which, even if target location is irrelevant to the task at hand, a behavioural advantage emerges when left- and right-side targets are responded to with a key placed on the left (vs. right) and on the right (vs. left), respectively. At a more conceptual level, numerous studies have also reported that centrally placed numbers are responded to faster with a key placed on the left (vs. right) side when they are relatively small (e.g., 1 or 2), whereas they are responded to faster with a key placed on the right (vs. left) side when they are relatively large (e.g., 8 or 9), in line with the notion of a hypothetical left-right mental number line (Dehaene et al., 1993). Intriguingly, a similar effect can also emerge for nonnumerical magnitudes (for a review see Macnamara et al., 2018).

Of particular relevance for the present work, several studies have also reported a left-right spatial representation of the valence associated with a stimulus. Specifically, more negatively valenced stimuli would be associated with the left part of space, whereas those connected with a more positive valence would be associated with the right part of space, likely reflecting a space-valence association (Giuliani et al., 2021; Pitt \& Casasanto, 2018; Root et al., 2006). For instance, in the study by Pitt and Casasanto (2018), participants were involved in a speeded binary classification task to establish whether a centrally placed word conveyed either a negative (e.g., 'horrible') or a positive (e.g., 'perfect') meaning. The results showed faster latencies when the negative and positive words were responded to with a leftside key and a right-side key, respectively, rather than when the opposite response mapping (i.e., negative-right, positiveleft) was adopted. Furthermore, the left-right space-valence association would be deeply shaped by the handedness of the individuals. Indeed, whereas a left-negative/right-positive association would emerge among right-handers, the opposite association would emerge among left-handers, as documented by several works (Brunyé et al., 2012; Casasanto, 2009; Casasanto \& Chrysikou, 2011; De la Vega et al., 2012; Kong, 2013). According to Casasanto (2009), the opposite space-valence representation expressed by the two groups (i.e., left-handers vs. right-handers) would be caused by bodily characteristics (i.e., the so-called body-specificity hypothesis). In particular, one possible explanation for this difference assumes that individuals would tend to implicitly relate positive (vs. negative) concepts with the spatial location with which they are more (vs. less) proficient in interacting, such as their dominant (vs. non-dominant) side. Importantly, according to some evidence (de la Fuente, et al., 2015), the space-valence association appears to be consistent even across different cultures (e.g., Spanish vs. Moroccan individuals), thus excluding potential confounds due to habits or social norms (e.g., writing direction). It is worth noting that in addition to the motor origins pushed forward by Casasanto (2009), other researchers identified in hemispheric specialisation other potential bases for space-valence associations (for a review see Demaree, et al., 2005). More precisely, whereas the left hemisphere (involved in right-side processing) would be more strongly engaged by positive or approach-related stimuli, the right hemisphere (involved in left-side processing) would be more strongly engaged by negative or avoidance-related stimuli. Although the roots of space-valence associations are still a matter of debate, the handedness of the individuals appears to be an important factor to consider when assessing this phenomenon.

\section{The Present Work}

We devised a speeded binary classification task, based on left- and right-side responses, in which participants were asked to categorise facial stimuli as belonging to either own-race or other-race individuals. In doing so, two primary goals were set. The first goal of this work was to replicate the ORCA through a cross-cultural approach involving Asian Japanese and White Italian individuals, recruited and tested in their own country (i.e., Japan and Italy), and 
presented with both Asian and White faces. To the best of our knowledge, so far, no studies have compared these two nationalities. Therefore, this would provide a further demonstration of both the generalizability and stability of the ORCA. In line with previous literature (Ge et al., 2009; Levin, 1996; Woo et al., 2020), we expected to observe - in both groups - faster responses to other-race faces as compared with own-race faces. The second goal of this work was to assess whether a left-right space-valence association could also emerge for other-race vs. own-race faces. In this regard, it is well established that other-race and own-race individuals are typically associated with negative and positive valence, respectively (Brewer, 1999). More related to the current work, a seminal study (Dunham et al., 2006) has also reported the presence of a reliable in-group bias in both Asian Japanese individuals living in Japan and White individuals living in a Western country (i.e., the USA) when exposed to different social groups (i.e., Japanese, Whites, and Blacks). Importantly, evidence of an in-group bias has also been reported in a more recent study (Esposito et al., 2014) involving Asian Japanese individuals living in Japan and White Italians living in Italy, in which participants were asked to explicitly report their feelings toward pictures of in-group and out-group individuals. The results showed that both Japanese and Italians associated more positive feelings with in-group (vs. out-group) individuals. Here, given the apparent association between own-race (vs. other-race) individuals and positive (vs. negative) valence, faster responses were predicted when own- and other-race faces were responded to with a right- and a left-side key, respectively - as compared with the opposite mapping (i.e., own-race/left side, other-race/right side) - and this pattern was expected to be particularly evident among right-handers. If confirmed, this would suggest that a left-right space-valence association could also be revealed within a social dimension based on group affiliation, likely reflecting the different valence levels typically associated with in-group and out-group members.

\section{Experiment 1: Asian Japanese Sample}

\section{Participants}

The sample size required for both Experiments was established a priori through a power analysis. In particular, we followed the guidelines (Brysbaert \& Stevens, 2018) proposed when linear mixed-effects models with subjects and items as random factors are employed (see results). Specifically, a minimum of 1600 data points per experimental cell should be collected. In our case, we devised a paradigm based on 60 trials per experimental cell per participant, thus implying a minimum number of 27 participants. We stopped at $\mathrm{N}=31$ for convenience, at the end of a booking session.
All participants were naïve Asian Japanese students (Mean age $=20.97$ years, $S D=1.8,13$ males), with normal or corrected-to-normal vision, recruited at the Waseda University (Tokyo, Japan). The study was approved by the institutional review board of the Waseda University and was conducted in accordance with the Declaration of Helsinki. Informed, written consent was obtained from all participants.

As for participants' handedness, although the left-negative/right-positive association appears to be specific to right-handers (Casasanto, 2009), we decided not to apply any a priori exclusion of left-handers (who are, in any case, a minority in the population worldwide) to avoid any sampling bias and because a more heterogeneous sample - if any - is more representative of the general population and allows for greater generalisability of the results (Willems et al., 2014). For this reason, we also planned to perform analyses both on the whole sample recruited, and then - if left-handers were present - only considering right-handers, to obtain a more suitable sample for testing the body-specificity hypothesis (Casasanto, 2009). We anticipate here that, for the sake of clarity, the analyses performed on righthanders - which were of primary importance - are reported in the main text, whereas the analyses for the whole sample are in dedicated footnotes. In the present sample, all participants self-declared to be right-handed. A more reliable and precise index of manual preference was also collected by asking participants to fill out the Edinburgh Handedness Inventory (EHI), short version (Veale, 2014). This tool, consisting of four items assessing the preferences in the use of hands for some activities and objects (e.g., writing or using the spoon), provides a score on a scale ranging from -100 , indicating an absolute preference for the left hand, to +100 , indicating an absolute preference for the right hand. The mean EHI score observed in this sample was +78 $(S D=41.83$; mode $=+100$; range: -88 to +100$)$. More specifically, according to the EHI classification scale (Veale, 2014), one participant was categorised as a left-hander (i.e., EHI score $=-88$ ), two participants as mixed handers (i.e., EHI scores $=-50,+50)$, and the remaining participants as right-handers (i.e., EHI mean score $=+90 ; S D=12.29$; mode $=+100$; range: +63 to +100$)$. The sample of righthanders was therefore composed of 28 individuals (Mean age $=21.04$ years, $S D=1.8,11$ males), which still guarantees adequate statistical power (according to the power analysis, $\mathrm{N}=27$ was the minimum sample size).

\section{Stimuli, Apparatus, and Procedure}

The stimuli consisted of photographs of 20 faces belonging to Asian models (10 males and 10 females) and 20 faces belonging to White models (10 males and 10 females). All models were young adults (age range: $21-31$ years). The photos were taken in a room with controlled lighting, with 
Fig. 1 Examples of trials in which an Asian face (A) and a White face (B) are depicted. The green ' $\mathrm{O}$ ' and the red ' $\mathrm{X}$ ' were the visual feedbacks indicating correct vs. wrong/ missing responses, respectively. Stimuli are not drawn to scale. Note: For illustrative purposes only, faces provided by the free access MR2 database (Strohminger et al., 2016) are shown

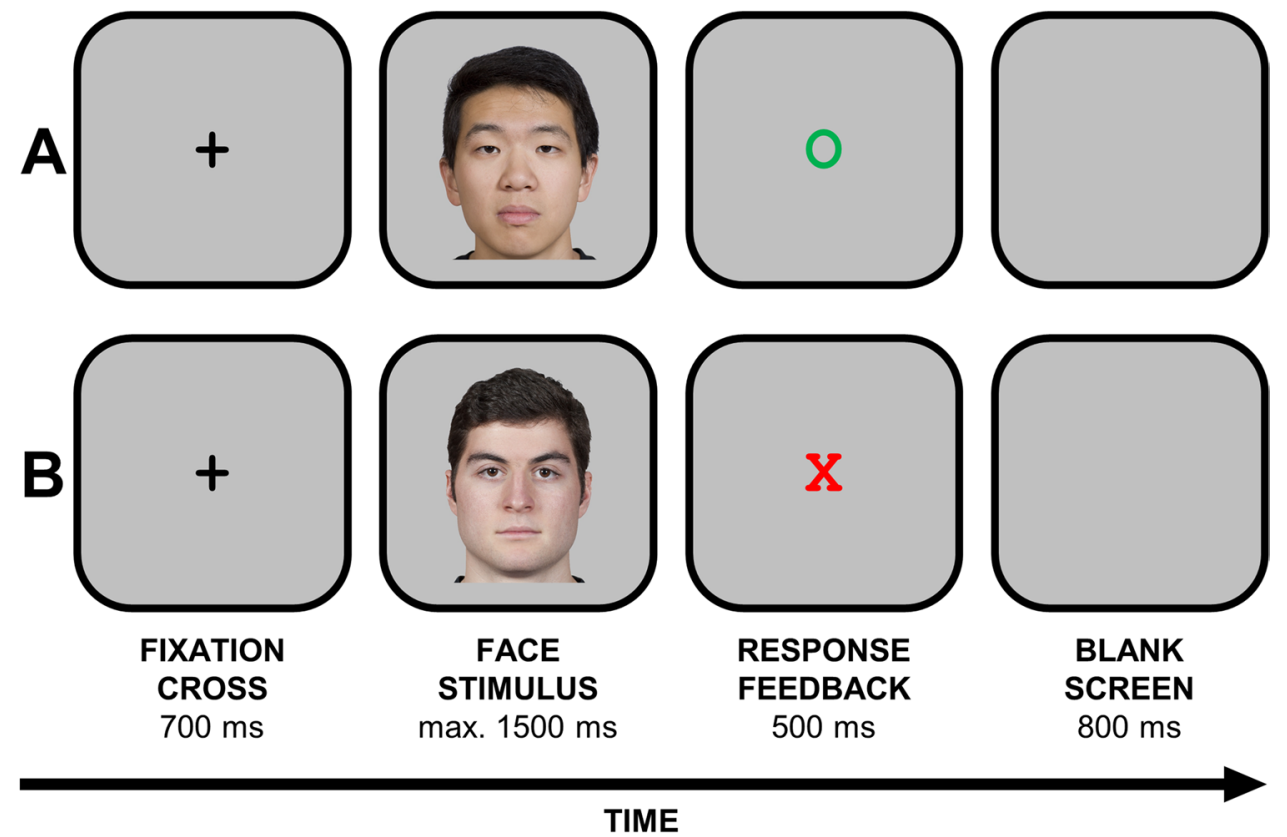

TIME the model wearing a black T-shirt and displaying a neutral facial expression (Nakamura et al., 2019). Stimuli were presented through E-Prime 2.0 on a PC monitor $(1920 \times 1080$ px, $60 \mathrm{~Hz}$ ) placed $57 \mathrm{~cm}$ from the participant. The screen background was set to silver colour $(R=192, G=192$, $B=192$ ). Manual responses were collected through a standard keyboard placed centrally in front of the PC monitor.

All participants were tested, in a dimly illuminated room, by an Asian Japanese experimenter. Examples of trials are depicted in Fig. 1. Both the task and the relevant parameters (e.g., timing of the stimuli) were similar to those used in previous studies conducted in analogue research (see, e.g., Dalmaso \& Vicovaro, 2019; Holmes \& Lourenco, 2011; Prete, 2020). Each trial started with a black fixation cross (side: $1^{\circ}$ ) presented at the centre of the screen for $700 \mathrm{~ms}$, followed by a central facial stimulus (about $8^{\circ}$ width $\times 12^{\circ}$ height). Participants were asked to classify - as fast and accurately as possible - the face as belonging to either an Asian or a White individual by pressing either a left-side key (i.e., Q) with the left index finger or a right-side key (i.e., P) with the right index finger. Importantly, the left-side key was pressed with the left hand, and the right-side key with the right hand. They were also instructed to look at the centre of the screen for the duration of the trial. The face remained visible until a response was detected or $1500 \mathrm{~ms}$ had elapsed, whichever came first. Then, central visual feedback appeared for $500 \mathrm{~ms}$, consisting of a green ' $\mathrm{O}$ ' ( side: $1^{\circ}$ ) in case of a correct response and a red ' $\mathrm{X}$ ' ( side: $\left.1^{\circ}\right)$ in case of either a wrong or a missed response. Finally, a blank screen was presented for $800 \mathrm{~ms}$. There were two practice blocks composed of 10 trials each (i.e., 20 practice trials in total), both followed by an experimental block of 120 trials (i.e., 240 experimental trials in total). As for the two experimental blocks, all trials were presented randomly and an equal number of times. The association between face ethnicity (i.e., White vs. Asian) and response side (i.e., left vs. right) was inverted in the two blocks; namely, in one block, White faces were responded to with the left key and Asian faces with the right key, whereas in the other block, the opposite occurred. Block order was counterbalanced across participants. At the end of the experimental session, participants were asked 1) to complete a computerised version of the EHI and 2) to identify themselves as 'Asian', 'White', or 'Other'. In both cases, participants were asked to provide their response by pressing a numeric key with no time limit.

\section{Results}

\section{Data Handling}

All participants self-identified as Asian individuals, and therefore they were all included in subsequent analyses. Both wrong (3.15\% of trials) and missed ( $0.78 \%$ of trials) responses were discarded and not further analysed due to their low percentage. Correct-response trials with a reaction time (RT) smaller or greater than 3 standard deviations below or above each participant's mean were considered outliers and discarded as well (1.62\% of trials).

\section{Analyses of Right-Handed Participants}

The RTs of correct trials were analysed with a linear mixed model implemented in $\mathrm{R}$ (https://cran.r-project. org/) through the lme4 package. We performed a likelihood 


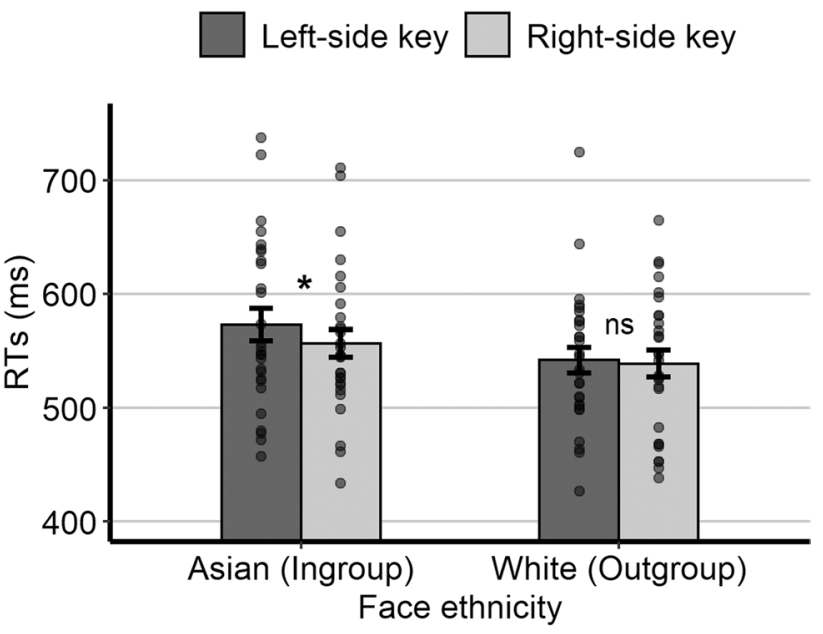

Fig. 2 Mean RTs, and individuals scores, observed among righthanders of Experiment 1 (Asian Japanese sample). Error bars are SEM. $*=p<.05 ; \mathrm{ns}=$ non-significant. Please note that, for each of the four cells of the experimental design, all individual scores fell within 3 standard deviations from the group mean

ratio test comparing models characterised by increasing complexity (i.e., from the null model to the saturated one). The best model fitting our data included face ethnicity (2: Asian vs. White), response side (2: left vs. right), and their interaction as fixed effects, while the random effects were the intercepts for subjects and face stimuli (i.e., the 40 different identities) and the by-subject slope for the effects of face ethnicity and response side. This model was then submitted to a Type 1 ANOVA (Satterthwaite's approximation for degrees of freedom) for linear mixed-effects models implemented through the lmerTest package. The main effect of face ethnicity was statistically significant, $F(1,48.3)=11.081, p=0.002$, because White faces $(M=541 \mathrm{~ms}, S E=11.5)$ elicited faster responses than Asian faces $(M=565 \mathrm{~ms}, S E=12.8)$, thus confirming the presence of the ORCA (Ge et al., 2009; Levin, 1996; Woo et al., 2020). The main effect of response side was also statistically significant, $F(1,27.2)=5.76, p=0.024$, because right-side responses $(M=548 \mathrm{~ms}, S E=11.3)$ were faster than left-side responses $(M=558 \mathrm{~ms}, S E=12.1)$. Importantly, the face ethnicity $\times$ response side interaction was also significant, $F(1,6265.4)=5.792, p=0.016$. Tukey's HSD planned comparisons for linear mixed-effects models, implemented through the lsmeans package, showed that, for White faces, right-side responses $(M=539 \mathrm{~ms}, S E=11.4)$ and left-side responses $(M=542 \mathrm{~ms}, S E=12.0)$ were not statistically different $(p=0.485)$, whereas for Asian faces, right-side responses $(M=557 \mathrm{~ms}, S E=12.5)$ were faster $(p<0.001)$ than left-side responses $(M=573 \mathrm{~ms}$,
$S E=13.4$; see also Fig. 2; for the analyses of the whole sample see the footnote ${ }^{1}$ ).

\section{Experiment 2: White Italian sample}

In the second experiment, everything was identical to Experiment 1 , with the only exception being that both the participants and the experimenter were White Italian individuals living in Italy.

\section{Participants}

As in Experiment 1, we aimed to recruit at least 27 individuals. We stopped at $\mathrm{N}=34$ for convenience, at the end of a booking session. All participants were naïve White Italian students (Mean age $=21.38$ years, $S D=1.28,6$ males) with normal or corrected-to-normal vision, recruited at the University of Padova (Padova, Italy). The study was approved by the Ethics Committee for Psychological Research of the University of Padova and conducted in accordance with the Declaration of Helsinki. Informed, written consent was obtained from all participants.

Regarding participants' handedness, three participants declared to be left-handers, and the mean EHI score was +65 $(S D=48.51$; mode $=+88$; range: -100 to +100$)$. More specifically, according to the EHI classification scale (Veale, 2014), one participant was categorised as a left-hander (i.e., EHI score $=-100$ ), four participants as mixed handers (i.e., EHI score $=-50,-50,-38,+50$ ), and the remaining participants as right-handers (i.e., EHI mean score $=+82$; $S D=12.73 ;$ mode $=+88$; range: +63 to +100$)$. The sample of right-handers was therefore composed of 29 individuals (Mean age $=21.28$ years, $S D=1.07,4$ males), which guarantees adequate statistical power.

\footnotetext{
${ }^{1}$ Data from the whole sample were analysed in the same manner as the data for right-handed participants. The main effect of face ethnicity was statistically significant, $F(1,47.3)=12.604, p<.001$, because White faces $(M=541 \mathrm{~ms}, S E=10.8)$ elicited faster responses than Asian faces $(M=566 \mathrm{~ms}, S E=11.9)$. The main effect of response side approached the canonical level of significance, $F(1,30.0)=3.613$, $p=.067$, because right-side responses $(M=550 \mathrm{~ms}, S E=10.6)$ tended to be faster than left-side responses $(M=557 \mathrm{~ms}, S E=11.3)$. The face ethnicity $\times$ response side interaction was not significant, $F(1$, $6905.6)=0.034, p=.853$. Despite the non-significance of the interaction, for the sake of comparison with the results for right-handed participants, the interaction was further explored with planned comparisons. These confirmed that, for White faces, right-side responses $(M=537 \mathrm{~ms}, S E=10.8)$ and left-side responses $(M=545 \mathrm{~ms}$, $S E=11.3)$ were not statistically different $(p=.091)$, and the same was true for Asian faces, because right-side responses $(M=562 \mathrm{~ms}$, $S E=11.8)$ were not statistically different $(p=.139)$ from left-side responses $(M=569 \mathrm{~ms}, S E=12.4)$.
} 


\section{Apparatus, Stimuli, and Procedure}

Everything was identical to Experiment 1.

\section{Results}

\section{Data Handling}

Data were handled as in Experiment 1. In particular, all participants self-identified as White individuals, and therefore they were all included in subsequent analyses. Both wrong (4.14\% of trials) and missed ( $0.66 \%$ of trials) responses were discarded and not further analysed due to their low percentage, as well as RT outliers $(2.14 \%$ of trials).

\section{Analyses of Right-Handed Participants}

Data were analysed as in Experiment 1. The main effect of face ethnicity was statistically significant, $F(1,46.0)=9.179$, $p=0.004$, because Asian faces $(M=586 \mathrm{~ms}, S E=11.5)$ elicited faster responses than White faces $(M=607 \mathrm{~ms}$, $S E=11.6$ ), thus confirming the presence of the ORCA (Ge et al., 2009; Levin, 1996; Woo et al., 2020). The main effect of response side was also significant, $F(1,27.5)=7.542$, $p=0.011$, because right-side responses $(M=591 \mathrm{~ms}$, $S E=11.5)$ were faster than left-side responses $(M=602 \mathrm{~ms}$, $S E=11.0)$. More importantly, the face ethnicity $\times$ response side interaction was also significant, $F(1,6349.6)=11.333$, $p<0.001$. Planned comparisons showed that, for White faces, right-side responses $(M=606 \mathrm{~ms}, S E=12.1)$ and left-side responses $(M=607 \mathrm{~ms}, S E=11.8)$ were not statistically different $(p=0.753)$, whereas for Asian faces, right-side responses $(M=575 \mathrm{~ms}, S E=12.1)$ were faster $(p<0.001)$ than left-side responses $(M=597 \mathrm{~ms}, S E=11.5$; see also Fig. 3; for the analyses of the whole sample see the footnote ${ }^{2}$.

\footnotetext{
${ }^{2}$ Data from the whole sample were analysed in the same manner as adopted for right-handed participants. The main effect of face ethnicity was statistically significant, $F(1,46.7)=11.862, p=.001$, because Asian faces $(M=593 \mathrm{~ms}, S E=11.8)$ elicited faster responses than White faces $(M=615 \mathrm{~ms}, S E=12.0)$. The main effect of response side was also significant, $F(1,32.5)=10.972, p=.002$, because right-side responses $(M=597 \mathrm{~ms}, S E=11.5)$ were faster than leftside responses $(M=611 \mathrm{~ms}, S E=11.8)$. More importantly, the face ethnicity $\times$ response side interaction was also significant, $F(1$, $7466.3)=11.328, p<.001$. Planned comparisons showed that, for White faces, right-side responses $(M=613 \mathrm{~ms}, S E=12.1)$ and leftside responses $(M=617 \mathrm{~ms}, S E=12.5)$ were not statistically different $(p=.391)$, whereas for Asian faces, right-side responses $(M=582 \mathrm{~ms}$, $S E=12.0)$ were faster $(p<.001)$ than left-side responses $(M=605 \mathrm{~ms}$, $S E=12.1)$
}

Left-side key $\square$ Right-side key

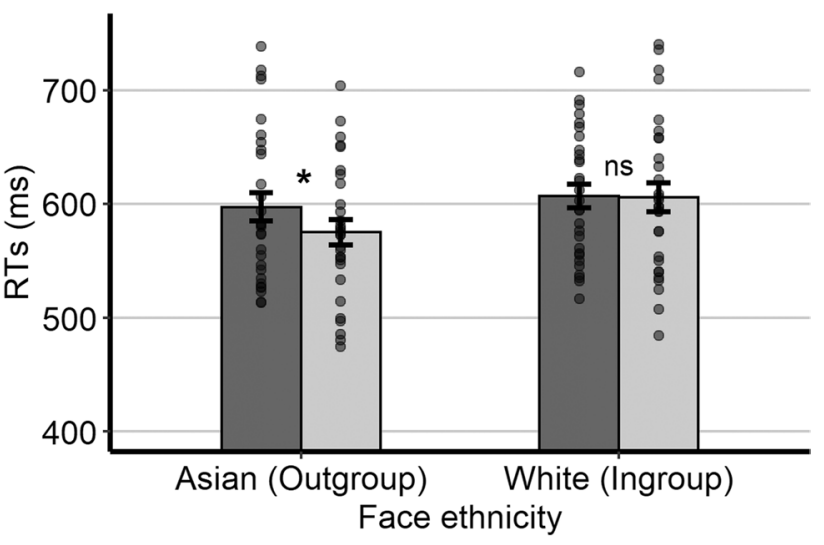

Fig. 3 Mean RTs, and individuals scores, observed among righthanders of Experiment 2 (White Italian sample). Error bars are SEM. $*=p<.05$; ns $=$ non-significant. Please note that, for each of the four cells of the experimental design, all individual scores fell within 3 standard deviations from the group mean

\section{Discussion}

The aim of this work was twofold. First, we wanted to provide further supporting evidence for the ORCA (Levin, 1996, 2000; Valentine \& Endo, 1992). Second, we wanted to explore whether a left-right space-valence association could be reported for other-race and own-race faces. To properly address these two goals, we adopted a cross-cultural approach involving both Asian Japanese (Experiment 1) and White Italian (Experiment 2) individuals living in Japan and Italy, respectively. Both groups completed a speeded binary classification task requiring them to categorise a centrally placed face as either Asian or White. Manual responses were provided through a left-side and a right-side response key.

As for the first aim, we found that the ORCA emerged in both experiments. Indeed, whereas Asian Japanese individuals (Experiment 1) were faster in categorising a facial stimulus as White (other race) rather than Asian (own race), the opposite pattern occurred among White Italian individuals (Experiment 2), because responses were faster for Asian faces (other race) than for White faces (own race). Taken together, these results confirm what was reported in previous studies (Ge et al., 2009; Levin, 1996; Woo et al., 2020) and extend the ORCA to a novel social comparison. Indeed, the new combination of ethnic memberships (i.e., Japanese vs. Italian) adds further evidence to the validity of the ORCA. The results of our experiments provide further confirmation of the robustness and generalizability of this - still scarcely investigated - phenomenon underlying face processing.

As for the second aim, an increasing number of studies have suggested that, among right-handers, stimuli with a negative and a positive valence would be associated with the 
left and the right side of the space, respectively, whereas the opposite space-valence mapping would emerge among lefthanders (Brunyé et al., 2012; Casasanto, 2009; De la Vega et al., 2012). In the present context, we expected that ownand other-race faces would be responded to faster with the right-side key than with the left-side key, as compared with the opposite mapping (i.e., own-race/left-side, other-race/rightside). This was specifically expected when data provided by right-handers (the majority group in both experiments) were considered (Casasanto, 2009). If confirmed, this pattern of results would have provided supporting evidence for the notion that in-group and outgroup can be spatially connoted, likely reflecting the different valence levels typically associated with the two groups. Importantly, the main finding here was that a space-ethnicity association clearly emerged in both Experiments 1 and 2, as confirmed by the significant interactions between face ethnicity and response side. Interestingly, this association was similar in both experiments. In more detail, on the one hand, Asian faces were responded to faster with the right-side key than with the left-side key, whereas, on the other hand, responses to White faces were unaffected by response side, with practically no differences in reaction times associated with the two keys. Whereas faster responses to Asian faces with the right-side key than the left-side key were predicted among Japanese individuals, they were unexpected among Italians because, according to previous evidence, both Asian Japanese individuals living in Japan and White individuals living in Western countries - Italy included - would tend to display, when exposed to different ethnic groups (i.e., Japanese, Whites, and Blacks), a reliable in-group bias (Dunham et al., 2006; Esposito et al., 2014). Here, we can tentatively propose that the pattern of results emerging among Italians might reflect specific factors characterising the context in which the study was conducted (i.e., Italy). In this regard, a previous study conducted among White Italians living in Italy provided supporting evidence for a preference towards Japanese outgroup members than Italian in-group members, likely reflecting a relatively high competence and social status attributed by Italians to Japanese (Vaes et al., 2010). In light of these findings, the results of our two experiments might indicate that Japanese individuals were positively connoted in both participant samples. ${ }^{3}$ Beyond this speculative conclusion,

\footnotetext{
${ }^{3}$ One reviewer wondered if attractiveness differed between Asian and White faces. For this reason, we delivered an online questionnaire, created with Qualtrics, to a new sample of Asian Japanese individuals living in Japan $(\mathrm{N}=20$; Mean age $=25.7$ years, $S D=4.46$, 11 males) and to a new sample of White Italian individuals living in Italy $(\mathrm{N}=21 ;$ Mean age $=23.1$ years, $S D=2.41,9$ males $)$. Both samples were recruited within the same populations from which we sampled the participants of Experiments 1 and 2. The questionnaire consisted of the random presentation of the 40 facial stimuli used in Experiments 1 and 2. Each face appeared in the centre of the screen, and the participants rated it on a 7 -point scale $(1=$ 'Not at all attractive', $7=$ 'Extremely attractive') which appeared below the face.
}

we must acknowledge that two main sources of limitations associated with the present set of experiments invite caution in interpreting the results. Indeed, we did not include any specific measure assessing how Asian Japanese and White Italians were actually perceived, in terms of valence, within our two samples of respondents. Moreover, participants were exposed to both Asian and White facial stimuli without any further specific information concerning the potential nationality of the depicted individuals. Consequently, we cannot establish if, during the task, our respondents associated each of the two face groups with specific nationalities. Future studies are therefore needed to address these two issues more properly.

Referring back to the experimental results, it is also worth emphasising that responses to White faces were unaffected by response side, with practically no differences in reaction times between the left and the right key. In this regard, we note that the unbalanced response patterns between left-side and right-side responses are not so rare in speeded binary classification tasks involving non-numeric dimensions, such as loudness (Chang \& Cho, 2015), size (Ren, et al., 2011), and weight (Dalmaso \& Vicovaro, 2019). More precisely, a consistent finding among all the aforementioned studies is a reliable association between larger magnitudes and right-side responses, and a weaker-to-null difference for smaller magnitudes and left-side responses. Crucially, a recent work (Giuliani et al., 2021) reported a similar pattern of results also for valence, with a reliable association between positive valence stimuli and right-side responses, but a lack of association between negative valence stimuli and left-side responses. This pattern of results closely resembles what occurred in the present context if we assume that, in both experiments, Asian faces were associated with positive valence and White faces with neutral or negative valence. So far, no studies have been carried out with the specific purpose of providing potential explanations for the unbalanced response pattern characterising many different speeded binary classification tasks. Nevertheless, according to Giuliani and colleagues (Giuliani et al., 2021), it might be that non-numerical dimensions (valence included) are processed more indirectly and less uniquely than numbers, thus leading to weaker associations between space and the

Footnote 3 (continued)

Responses were provided, without time limits, with the mouse. As for the Japanese respondents, a two-tailed paired t-test showed that White faces $(M=4.04, S E=0.122)$ were rated more attractive than Asian faces $(M=3.71, S E=0.122 ; t(19)=2.664, p=.015, d=0.596)$. The same pattern of results also emerged within the Italian sample (White faces: $M=3.15, \quad S E=0.199$; Asian faces: $M=2.69, \quad S E=0.192$; $t(20)=4.414, p<.001, d=0.963$; for similar results, see also Rhodes et al., 2005). Even if this is indirect evidence, it indicates that the results obtained in Experiments 1 and 2 are unlikely to be driven by attractiveness (if this were the case, then the opposite results would have emerged from the questionnaire). 
manipulated dimension. The same rationale could therefore also be applied, prudently, to the present context.

Interestingly, whereas in Experiment 1 the space-ethnicity association emerged only after the removal of both left- and mixed-handers from the analyses, a comparable space-valence association emerged in Experiment 2 irrespective of whether the analyses involved the whole sample of participants or the subsample of right-handers. However, because EHI scores categorised only one participant per group as a left-hander, any specific consideration concerning the role of handedness in shaping our results is prevented. For this reason, in the next future it will be important to address more directly the potential impact of handedness by comparing the performance of right-handers with that of a similar group of left-handers, for which an opposite space-ethnicity association is expected. Analogously, according to some previous studies (Rodway et al., 2003; Van Strien \& Van Beek, 2000), the associations between space and face stimuli appear to be shaped even by the respondent's gender. ${ }^{4}$ Hence, additional future works could also include an equal number of male and female participants. Overall, this approach will allow for uncovering the precise influence of these two individual differences (i.e., handedness and gender) in the task adopted here.

So far, only a few studies explored the possible association that can occur between the valence conveyed by a given facial feature and the physical space. Most of these works employed faces displaying different expressions, such as happiness or angriness (see, e.g., Fantoni et al., 2019; Holmes \& Lourenco, 2011; Pitt \& Casasanto, 2018; Prete, 2020). These showed that - at least under specific circumstances - emotional valence (and its intensity) can be mapped onto the horizontal space, with negative (and lower-intensity) stimuli that would be mapped on the left and positive (and higher-intensity) stimuli that would be mapped on the right. Another recent study (Dalmaso \& Vicovaro, 2021) focused on face age and presented participants with relatively young and old faces, but no evidence of a spatial representation of this social dimension emerged. Overall, the scarcity of studies employing faces, along with the great relevance of faces for human cognition (see, e.g., Little et al., 2011), can be seen as an incentive to push future research on the space-valence association towards such a direction.

In recent years, flourishing literature has emerged reporting how cross-cultural differences can impact face processing and, more generally, the elaborations of social stimuli belonging to different ethnic groups (e.g., Caldara, 2017). In

\footnotetext{
${ }^{4}$ We also performed, for exploratory purposes, additional analyses in which the gender of the respondent was included in the models used to analyse the data of Experiments 1 and 2. In both Experiments, the crucial face ethnicity $\times$ response side interaction was still statistically significant $(p \mathrm{~s}<.016)$, but it was not further qualified by gender ( $p$ s $>$.456)
}

this regard, recent works found differences among different ethnic groups tested in their own countries in a number of behaviours supporting social interactions, such as face scanning during real social interaction (Haensel et al., 2020) or gaze-mediated orienting of attention (Zhang et al., 2021). Hence, the variety of methods and scientific backgrounds - deriving from different fields within psychology (e.g., cognitive, social) - allows cross-cultural studies to deeply explore different mechanisms underlying social perception under multiple perspectives. As a next step, it will be important to assess whether a space-ethnicity association can be also revealed by employing different face data sets by including other social groups (e.g., Blacks) and/or by focusing on different behavioural measures, such as eye movements (Gozli et al., 2013), and spatial dimensions, as up-down locations (e.g., Vicovaro \& Dalmaso, 2021), thus obtaining a broader view of this phenomenon.

\section{Conclusion}

To conclude, in two experiments, we administered a speeded binary classification task in which Japanese and Italian participants categorised a central face as belonging to either their own or another race. Overall, our results confirmed the presence of a reliable ORCA in both experiments. Moreover, we also obtained supporting evidence for a possible spaceethnicity association. Interestingly, this was similar in both samples, which could suggest analogous attitudes towards the two ethnic groups involved. However, it is worth noting that this latter conclusion is speculative and awaits further experimental studies. Taken together, these findings may offer novel insights regarding the mechanisms supporting social perception, categorisation, and their interplay, as seen from a cross-cultural point of view.

Acknowledgements We are grateful to Koyo Nakamura for valuable information concerning the face database used here. We also thank two undergraduate students for their assistance with data collection.

Authors' Contributions Mario Dalmaso (Conceptualization; Data curation; Formal analysis; Prepared the figures; Supervision; Writing - original draft; Writing - review \& editing) Michele Vicovaro (Conceptualization; Data curation; Formal analysis; Supervision; Writing - review \& editing) Katsumi Watanabe (Conceptualization; Resources; Supervision; Writing - review \& editing).

Funding This work was partially supported by a mobility grant (Fondi Area Relazioni Internazionali, Accordi Bilaterali 2019, University of Padova) and a departmental grant (DPSS-SID2019) both awarded to MD, by a grant from MIUR (Dipartimenti di Eccellenza DM 11/05/2017 n. 262) to the Department of General Psychology, University of Padova, and by a grant-in-aid for Scientific Research on Innovative Areas (17H06344) from JSPS awarded to KW.

Data Availability Raw experimental data and questionnaire data are available in the OSF repository at $10.17605 / O S F . I O / Z H G 3 F$. 
Code Availability The experiment code is available in the OSF repository at $10.17605 / \mathrm{OSF} . \mathrm{IO} / \mathrm{ZHG} 3 \mathrm{~F}$.

\section{Declarations}

Ethics Approval All methods were approved by the "Institutional Review Board" of Waseda University and by the "Ethics Committee for Psychological Research" of the University of Padova. All methods were performed in accordance with the relevant guidelines and regulations and with the Declaration of Helsinki.

Consent to Participate Informed, freely-given, written consent was obtained from all participants.

Consent for Publication All participants consented to publish their data anonymously.

Conflicts of Interest/Competing Interests Financial interests: The authors declare they have no financial interests.

Non-financial interests: None.

Open Access This article is licensed under a Creative Commons Attribution 4.0 International License, which permits use, sharing, adaptation, distribution and reproduction in any medium or format, as long as you give appropriate credit to the original author(s) and the source, provide a link to the Creative Commons licence, and indicate if changes were made. The images or other third party material in this article are included in the article's Creative Commons licence, unless indicated otherwise in a credit line to the material. If material is not included in the article's Creative Commons licence and your intended use is not permitted by statutory regulation or exceeds the permitted use, you will need to obtain permission directly from the copyright holder. To view a copy of this licence, visit http://creativecommons.org/licenses/by/4.0/.

\section{References}

Adams, R. B., Ambady, N., Macrae, C. N., \& Kleck, R. E. (2006). Emotional expressions forecast approach-avoidance behavior. Motivation and Emotion, 30(2), 179-188. https://doi.org/10.1007/ s11031-006-9020-2

Brewer, M. B. (1999). The psychology of prejudice: Ingroup love or outgroup hate? Journal of Social Issues, 55(3), 429-444. https:// doi.org/10.1111/0022-4537.00126

Brunyé, T. T., Gardony, A., Mahoney, C. R., \& Taylor, H. A. (2012). Body-specific representations of spatial location. Cognition, 123(2), 229-239. https://doi.org/10.1016/j.cognition.2011.07.013

Brysbaert, M., \& Stevens, M. (2018). Power analysis and effect size in mixed effects models: A tutorial. Journal of Cognition, 1(1), 1-20. https://doi.org/10.5334/joc. 10

Caldara, R. (2017). Culture reveals a flexible system for face processing. Current Directions in Psychological Science, 26(3), 249-255. https://doi.org/10.1177/0963721417710036

Casasanto, D. (2009). Embodiment of abstract concepts: Good and bad in right- and left-handers. Journal of Experimental Psychology: General, 138(3), 351-367. https://doi.org/10.1037/a0015854

Casasanto, D., \& Chrysikou, E. G. (2011). When left is "Right": Motor fluency shapes abstract concepts. Psychological Science, 22(4), 419-422. https://doi.org/10.1177/0956797611401755

Chang, S., \& Cho, Y. S. (2015). Polarity correspondence effect between loudness and lateralized response set. Frontiers in Psychology, 06(May), 1-15. https://doi.org/10.3389/fpsyg.2015.00683
Dalmaso, M., Castelli, L., \& Galfano, G. (2020). Social modulators of gaze-mediated orienting of attention: A review. Psychonomic Bulletin \& Review, 27(5), 833-855. https://doi.org/10.3758/ s13423-020-01730-x

Dalmaso, M., \& Vicovaro, M. (2019). Evidence of SQUARC and distance effects in a weight comparison task. Cognitive Processing, 20(2), 163-173. https://doi.org/10.1007/s10339-019-00905-2

Dalmaso, M., \& Vicovaro, M. (2021). Is face age mapped asymmetrically onto space? Insights from a snarc-like task. Symmetry, 13(9), 1617. https://doi.org/10.3390/sym13091617

de la Fuente, J., Casasanto, D., Román, A., \& Santiago, J. (2015). Can culture influence body-specific associations between space and valence? Cognitive Science, 39(4), 821-832. https://doi.org/ $10.1111 / \operatorname{cogs} .12177$

De la Vega, I., de Filippis, M., Lachmair, M., Dudschig, C., \& Kaup, B. (2012). Emotional valence and physical space: Limits of interaction. Journal of Experimental Psychology: Human Perception and Performance, 38(2), 375-385. https://doi.org/10. 1037/a0024979

Dehaene, S., Bossini, S., \& Giraux, P. (1993). The mental representation of parity and number magnitude. Journal of Experimental Psychology: General, 122(3), 371-396. https://doi.org/10.1037/ 0096-3445.122.3.371

Demaree, H. A., Everhart, D. E., Youngstrom, E. A., \& Harrison, D. W. (2005). Brain lateralization of emotional processing: Historical roots and a future incorporating "dominance." Behavioral and Cognitive Neuroscience Reviews, 4(1), 3-20. https://doi.org/10. 1177/1534582305276837

Dunham, Y., Baron, A. S., \& Banaji, M. R. (2006). From american city to japanese village: A cross-cultural investigation of implicit race attitudes. Child Development, 77(5), 1268-1281. https://doi.org/ 10.1111/j.1467-8624.2006.00933.x

Esposito, G., Nakazawa, J., Ogawa, S., Stival, R., Kawashima, A., Putnick, D. L., \& Bornstein, M. H. (2014). Baby, you light-up my face: Culture-general physiological responses to infants and culture-specific cognitive judgements of adults. PLOS ONE, 9(10), 1-8. https://doi.org/10.1371/journal.pone.0106705

Fantoni, C., Baldassi, G., Rigutti, S., Prpic, V., Murgia, M., \& Agostini, T. (2019). Emotional semantic congruency based on stimulus driven comparative judgements. Cognition, 190, 20-41. https:// doi.org/10.1016/j.cognition.2019.04.014

Ge, L., Zhang, H., Wang, Z., Quinn, P. C., Pascalis, O., Kelly, D., ... Lee, K. (2009). Two faces of the other-race effect: Recognition and categorisation of Caucasian and Chinese faces. Perception, 38(8), 1199-1210. https://doi.org/10.1068/p6136

Giuliani, F., Manippa, V., Brancucci, A., Palumbo, R., Tommasi, L., $\&$ Pietroni, D. (2021). How emotional is a banknote? The affective basis of money perception. Psychological Research, 85(8), 3010-3025. https://doi.org/10.1007/s00426-020-01457-3

Gozli, D. G., Chow, A., Chasteen, A. L., \& Pratt, J. (2013). Valence and vertical space: Saccade trajectory deviations reveal metaphorical spatial activation. Visual Cognition, 21(5), 628-646. https://doi. org/10.1080/13506285.2013.815680

Haensel, J. X., Danvers, M., Ishikawa, M., Itakura, S., Tucciarelli, R., Smith, T. J., \& Senju, A. (2020). Culture modulates face scanning during dyadic social interactions. Scientific Reports, 10(1), 1-11. https://doi.org/10.1038/s41598-020-58802-0

Haxby, J., Hoffman, E., \& Gobbini, M. (2000). The distributed human neural system for face perception. Trends in Cognitive Sciences, 4(6), 223-233. Retrieved from http://www.ncbi.nlm.nih.gov/pubmed/10827445

Holmes, K. J., \& Lourenco, S. F. (2011). Common spatial organization of number and emotional expression: A mental magnitude line. Brain and Cognition, 77(2), 315-323. https://doi.org/10.1016/j. bandc.2011.07.002 
Hugenberg, K., Young, S. G., Bernstein, M. J., \& Sacco, D. F. (2010). The categorization-individuation model: An integrative account of the other-race recognition deficit. Psychological Review, 117(4), 1168-1187. https://doi.org/10.1037/a0020463

Kong, F. (2013). Space-valence associations depend on handedness: Evidence from a bimanual output task. Psychological Research, 77(6), 773-779. https://doi.org/10.1007/s00426-012-0471-7

Levin, D. T. (1996). Classifying faces by race: The structure of face categories. Journal of Experimental Psychology: Learning Memory and Cognition, 22(6), 1364-1382. https://doi.org/10.1037/ 0278-7393.22.6.1364

Levin, D. T. (2000). Race as a visual feature: Using visual search and perceptual discrimination tasks to understand face categories and the cross-race recognition deficit. Journal of Experimental Psychology: General, 129(4), 559-574. https://doi.org/10.1037/ 0096-3445.129.4.559

Little, A. C., Jones, B. C., \& DeBruine, L. M. (2011). The many faces of research on face perception. Philosophical Transactions of the Royal Society b: Biological Sciences, 366, 1634-1637. https://doi. org/10.1098/rstb.2010.0386

Macnamara, A., Keage, H. A. D., \& Loetscher, T. (2018). Mapping of non-numerical domains on space: A systematic review and metaanalysis. Experimental Brain Research, 236(2), 335-346. https:// doi.org/10.1007/s00221-017-5154-6

Nakamura, K., Ticcinelli, V., Richoz, A.-R., Caldara, R., \& Watanabe, K. (2019). Universality and cultural diversity in facial expression [in Japanese]. In Japanese Psychonomic Society Annual Meeting.

Nickerson, R. S. (1972). Binary-classification reaction time: A review of some studies of human information-processing capabilities. Psychonomic Monograph Supplements, 4(17), 275-318.

Pitt, B., \& Casasanto, D. (2018). Spatializing emotion: No evidence for a domain-general magnitude system. Cognitive Science, 42(7), 2150-2180. https://doi.org/10.1111/cogs.12568

Prete, G. (2020). Spatializing emotions besides magnitudes: Is there a left-to-right valence or intensity mapping? Symmetry, 12(5), 775. https://doi.org/10.3390/sym12050775

Ren, P., Nicholls, M. E. R., Ma, Y., \& ye, \& Chen, L. (2011). Size matters: Non-numerical magnitude affects the spatial coding of response. PLoS ONE, 6(8), 4-9. https://doi.org/10.1371/journal. pone. 0023553

Rhodes, G., Lee, K., Palermo, R., Weiss, M., Yoshikawa, S., Clissa, P., ... \& Jeffery, L. (2005). Attractiveness of own-race, other-race, and mixed-race faces. Perception, 34(3), 319-340. https://doi.org/ $10.1068 / \mathrm{p} 5191$

Rodway, P., Wright, L., \& Hardie, S. (2003). The valence-specific laterality effect in free viewing conditions: The influence of sex, handedness, and response bias. Brain and Cognition, 53(3), 452-463. https://doi.org/10.1016/S0278-2626(03)00217-3

Root, J. C., Wong, P. S., \& Kinsbourne, M. (2006). Left hemisphere specialization for response to positive emotional expressions: A divided output methodology. Emotion, 6(3), 473-483. https://doi. org/10.1037/1528-3542.6.3.473

Simon, J. R., \& Rudell, A. P. (1967). Auditory S-R compatibility: The effect of an irrelevant cue on information processing. Journal of
Applied Psychology, 51(3), 300-304. https://doi.org/10.1037/ h0020586

Strohminger, N., Gray, K., Chituc, V., Heffner, J., Schein, C., \& Heagins, T. B. (2016). The MR2: A multi-racial, mega-resolution database of facial stimuli. Behavior Research Methods, 48(3), 1197-1204. https://doi.org/10.3758/s13428-015-0641-9

Tanaka, J. W., Kiefer, M., \& Bukach, C. M. (2004). A holistic account of the own-race effect in face recognition: Evidence from a crosscultural study. Cognition, 93(1), 1-9. https://doi.org/10.1016/j. cognition.2003.09.011

Vaes, J., Heflick, N. A., \& Goldenberg, J. L. (2010). "We are people": Ingroup humanization as an existential defense. Journal of Personality and Social Psychology, 98(5), 750-760. https://doi.org/ 10.1037/a0017658

Valentine, T., \& Endo, M. (1992). Towards an exemplar model of face processing: The effects of race and distinctiveness. The Quarterly Journal of Experimental Psychology Section A, 44(4), 671-703. https://doi.org/10.1080/14640749208401305

Van Strien, J. W., \& Van Beek, S. (2000). Ratings of emotion in laterally presented faces: Sex and handedness effects. Brain and Cognition, 44(3), 645-652. https://doi.org/10.1006/brcg.1999.1137

Veale, J. F. (2014). Edinburgh handedness inventory - short form: A revised version based on confirmatory factor analysis. Laterality, 19(2), 164-177. https://doi.org/10.1080/1357650X.2013.783045

Vicovaro, M., \& Dalmaso, M. (2021). Is 'heavy' up or down? Testing the vertical spatial representation of weight. Psychological Research, 85, 1183-1200. https://doi.org/10.1007/ s00426-020-01309-0

Willems, R. M., Van Der Haegen, L., Fisher, S. E., \& Francks, C. (2014). On the other hand: Including left-handers in cognitive neuroscience and neurogenetics. Nature Reviews Neuroscience, 15(3), 193-201. https://doi.org/10.1038/nrn3679

Woo, P. J., Quinn, P. C., Méary, D., Lee, K., \& Pascalis, O. (2020). A developmental investigation of the other-race categorization advantage in a multiracial population: Contrasting social categorization and perceptual expertise accounts. Journal of Experimental Child Psychology, 197, 104870. https://doi.org/10.1016/j.jecp. 2020.104870

Zhang, X., Dalmaso, M., Castelli, L., Fiorese, A., Lan, Y., Sun, B., Fu, S., \& Galfano, G. (2021). Social attention across borders: A cross-cultural investigation of gaze cueing elicited by same- and other-ethnicity faces. British Journal of Psychology, 112, 741762. https://doi.org/10.1111/bjop.12476

Zhao, L., \& Bentin, S. (2011). The role of features and configural processing in face-race classification. Vision Research, 51(23-24), 2462-2470. https://doi.org/10.1016/j.visres.2011.10.001

Publisher's Note Springer Nature remains neutral with regard to jurisdictional claims in published maps and institutional affiliations. 